\section{Axitinib überzeugt in Zweitlinientherapie}

Axitinib ist ein selektiver VEGFR-Inhibitor (VEGFR: vaskulärer Endothelwachstumsfaktor-Rezeptor) der 2. Generation. Eine aktuelle US-amerikanische Studie hat gezeigt, dass Axitinib das progressionsfreie Überleben (PFS) von Patienten mit Zytokin-refraktärem Nierenzellkarzinom gegenüber Sorafenib verlängert. Lancet 2011; 378: 1931-1939

\section{mit Kommentar}

In der Phase-III-Studie AXIS behandelten Brian Rini, Cleveland Clinic Taussing Cancer Institute/USA, und Kollegen 723 Patienten mit klarzelligem, metastasiertem Nierenzellkarzinom mit den Tyrosinkinase-Inhibitoren Axitinib oder Sorafenib in einer Zweitlinientherapie (1:1-randomisiert). Die Patienten erhielten Axitinib $2 x$ $5 \mathrm{mg} / \mathrm{Tag}$ (bei guter Verträglichkeit Option zur Dosissteigerung bis auf $2 \times 10 \mathrm{mg} / \mathrm{Tag}$ ) oder Sorafenib 2 x $400 \mathrm{mg} /$ Tag. Die Studie wurde unter maßgeblicher Beteiligung von Pfitzer Inc. durchgeführt. Stratifiziert wurde nach Allgemeinzustand (ECOG Performance Status 0-1) und Art der systemischen Vortherapie. Diese war in beiden Studienarmen bei jeweils 54\% der Patienten mit Sunitinib erfolgt, bei 35\% mit Zytokinen, bei $8 \%$ mit Bevacizumab und bei $3 \%$ mit Temsirolimus. Die Behandlung endete bei Krankheitsprogress, inakzeptabler Toxizität, Tod oder auf Patientenwunsch. Der primäre Endpunkt war das Progressionfreie Überleben (PFS). Sekundäre Endpunkte waren die Gesamtüberlebenszeit, die objektive Ansprechrate, die Ansprechdauer und die Progression.

\section{Axitinib wirksamer als Sorafenib bei gleicher Verträglichkeit $\nabla$}

Das PFS war im Axitinib-Arm signifikant länger als im Sorafenib-Arm (6,7 vs. 4,7 Monate; Hazard Ratio: 0,665; p <0,0001). Das PFS war bei Zytokin-vorbehandelten Patienten am deutlichsten erhöht $(12,1$ bei Axitinib vs. 6,5 Monate bei Sorafenib). Bei Patienten, die als Erstlinientherapie Sunitinib erhielten, verbesserte sich das PFS unter Axitinib nur wenig gegenüber Sorafenib (4,8 vs. 3,4 Monate). Für die Bevacizumab- und Temsirolimus-Gruppe waren die Ergebnisse aufgrund der geringen Fallzahl nicht aussagekräftig.

Bezüglich der sekundären Endpunkte war die objektive Ansprechrate unter Axitinib signifikant höher als unter Sorafenib (19 vs. 9\%) bei einer medianen Ansprechdauer von 11,0 (Axitinib) vs. 10,6 Monaten (Sorafenib). Die Rate der stabilen (27 unter Axitinib vs. $21 \%$ unter Sorafenib) und der progressiven Erkrankungen (22 vs. $21 \%)$ unterschied sich nicht wesentlich. Im Axitinib-Arm war die Zeitspanne bis

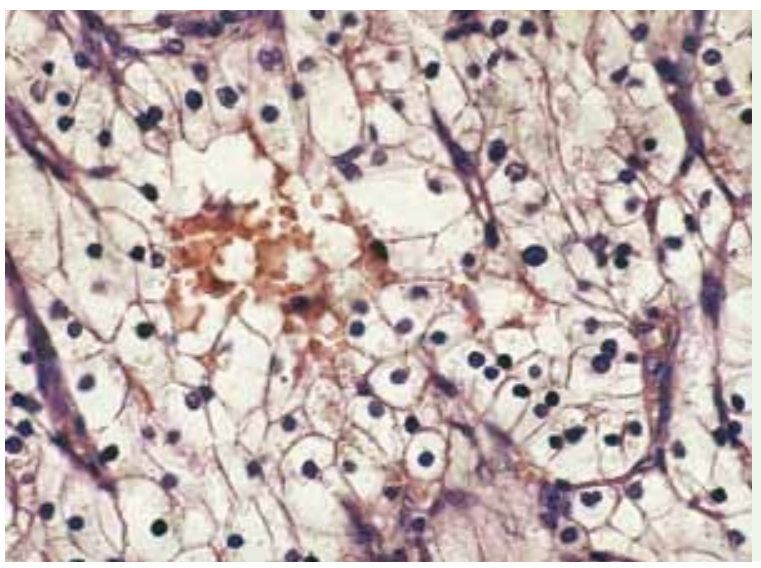

Nierenzellkarzinom, klarzelliger Typ (Vergr. 1:150). Laut der aktuellen Studie verlängerte Axitinib gegenüber Sorafenib das progressionsfreie Überleben von Patienten mit metastasiertem Nierenzellkarzinom um bis zu 6 Monate bei vergleichbarem Sicherheitsprofil (Bild: Riede U-N, Schäfer H-E, Werner M, aus: Allgemeine und spezielle Pathologie; Thieme 2004). zu einer Verschlechterung der Krankheit länger. Die mittlere Dosisintensität, definiert als Quotient von tatsächlicher und geplanter Gesamtdosis, lag bei $99 \%$ in der Axitinib- und bei $92 \%$ in der SorafenibGruppe. Aufgrund toxischer Nebenwirkungen wurde die Axitinib-Therapie bei $4 \%(n=14)$ und die Sorafenib-Therapie bei $8 \%$ der Patienten $(n=29)$ abgebrochen. Das Sicherheitsprofil beider Substanzen war vergleichbar. Bei mehr als $30 \%$ der Patienten traten unter Axitinib unerwünschte Nebenwirkungen auf, am häufigsten Diarrhö, Fatigue, Hypertonie, Appetitverlust, Übelkeit und Dysphonie. Die häufigsten Nebenwirkungen unter Sorafenib waren neben Diarrhö und Fatigue das HandFuß-Syndrom, Alopezie und Exantheme.

\section{Fazit}

Bei vergleichbarer Sicherheit führte Axitinib gegenüber Sorafenib in der aktuellen Studie zu einem längeren progressionsfreien Überleben und bietet somit eine weitere Option für die Zweitlinientherapie des metastasierten Nierenzellkarzinoms. Weitere Vergleichsstudien seien erforderlich, um die zielgerichtete Therapie des Nierenzellkarzinoms zu optimieren, so die Autoren.

Renate Ronge, Münster

Kommentar

Gutes Ansprechen auf Axitinib nach Tumorprogression

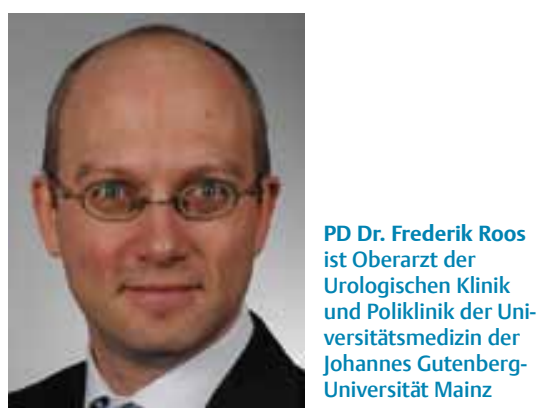

Kleinmolekulare Angiogeneseinhibitoren

In den letzten Jahren hat sich die Behandlung des metastasierten Nierenzellkarzinoms (mNZK) von immunologischen Therapieansätzen, die über die letzten 2 Jahrzehnte den Therapiestandard bestimmt haben, weitestgehend entfernt [1]. Nach dem besseren Verständnis der Tumorbiologie des NZK und der Identifi- 
kation der mit den zytogenetischen Alterationen in Verbindung stehenden Mutationen von Tumorsuppressor- und/oder Proto-Onkogenen konnten kleine Moleküle (zielgerichte Inhibitoren) synthetisiert werden, die gezielt in die Signaltransduktionswege der Tumorzelle eingreifen [2]. Insbesondere VEGF als potenter Wachstumsfaktor der Angiogenese des NZK bietet gute Möglichkeiten zur Entwicklung kleiner Moleküle, die in die VEGF-Signalkaskade eingreifen [3]. Hierzu zählen die bisher zugelassenen und in der klinischen Praxis verwendeten Thyrosinkinaseinhibtoren (TKI) Sunitinib, Sorafenib und seit Kurzem Pazopanib, die gezielt den VEGF-Rezeptor und gleichermaßen den PDGF-Rezeptor inhibieren [4]. Diese Therapieformen sind effektiv in der Behandlung des mNZK und mit einem insgesamt tolerablen Nebenwirkungsspektrum verbunden. Allerdings sind die Vorteile im Gesamtüberleben gegenüber den Kontrollarmen in den Phase-III-Studien größtenteils statistisch nicht signifikant [5] oder belaufen sich auf 2-4 Monate [6].

Zielgerichtete, patientenorientierte, individualisierte Therapie

Welche medikamentöse Therapie für welchen Patienten am besten geeignet ist, wird zurzeit in Subanalysen einiger klinischer Studien untersucht $[7,8]$. Hierbei ist es auch unerlässlich im Rahmen der patientenorientierten individualisierten Therapie auf die Tumorcharakteristika (z.B. histologischer Subtyp, sarkomatoide Differenzierung) und auf Patientenwünsche, Begleitmedikation und Komorbiditäten zu achten. Folglich sind mehrere Studien in der klinischen Anwendung, die Patientenakzeptanz und Nebenwirkungsspektrum zweier Angiogeneseinhibitoren testen [9, 10]. Einen direkten Vergleich zwischen zwei TKIs unternimmt aktuell die COMPARZ-Studie (Pazopanib vs. Sunitinib) untersucht [11]. Die Erfahrungen zeigen im Allgemeinen, dass es nach der Erstlinientherapie nach ca. 6 Monaten wieder zu einem Tumorprogress kommt und eine Zweitlinientherapie notwendig ist. Zur Erst- und Zweitlinientherapie sind Richtlinien von der EAU und der EORTCGU publiziert worden $[12,13]$.

\section{Sequenztherapie}

Eine Sequenztherapie für Patienten mit metastasiertem NZK in Richtung Drittund Viertlinientherapie gewinnt immer mehr an Bedeutung, da hierdurch der Tumorprogress über mehrere Monate weiter herausgezögert werden kann. Welche Sequenz (VEGF-Antikörper und/oder TKITKI-mTOR vs. TKI-mTOR-TKI) gewählt wird, ist von vielen Faktoren und v.a. vom Patienten selbst abhängig.

In dieser Vergleichsstudie wurde Axitinib, das die VEGFR 1-3 inhibiert, als Zweitgeneration TKI gegen Sorafenib getestet [14]. Eine interessante und wichtige Information birgt die Randomisierung, in der unterschiedlichste Erstlinientherapien berücksichtigt werden konnten. Hierbei ist erwähnenswert, dass das progressionsfreie Überleben unter Axitinib nach zytokin- und mTOR-basierter Erstlinientherapie fast doppelt so lang war, wie unter Sorafenib. Die Ergebnisse nach Therapie mit Bevacizumab und Sunitinib waren dagegen vergleichbar. Allerdings muss hierbei berücksichtigt werden, dass nur eine geringe Anzahl der Patienten mit Bevacizumab und Temsirolimus in der Erstlinientherapie behandelt wurde.

Eine 19\%ige gegenüber einer $9 \%$ igen objektiven Ansprechrate spricht für Axitinib, wobei die Dauer des Ansprechens mit 11 Monaten unter Axitinib vs. 10,6 Monaten unter Sorafenib fast identisch waren. Die Autoren sprechen von einem tolerablen und einem vergleichbarem Nebenwirkungsprofil unter Axitinib gegenüber Sorafenib. Genauer betrachtet ist der prozentuale Anteil der $\geq$ Grad-3-Nebenwirkungen für Nausea, Vomitus, Fatigue, Durchfall und Asthenie unter Axitinib größer als unter Sorafenib. Die Lebensqualität der Patienten unter den Therapeutika wurde nicht dokumentiert, was eine bedeutende Schwäche der Studie ist, da es für eine individualisierte, patientenorientierte Therapie unerlässlich ist, zu wissen für welchen alltagseinschränkenden Preis eine Progressionsverlängerung erkauft wird.

Wichtig ist die Erkenntnis, dass ein gutes Ansprechen auf Axitinib nach Tumorprogression unter einem mTOR-Inhibitor beobachtet werden konnte, was bei einigen Patienten für eine Resensibilisierung des Tumors gegenüber TKIs spricht [15]. Ob Axitinib in der Zweit- oder Drittlinientherapie verwendet wird, werden weitere Studien klären. Meineserachtens könnte Axitinib in der Sequenztherapie nach einem mTOR-Inhibitoren angewendet werden.

PD Dr. Frederik Roos, Mainz 
Literatur

1 Tong TQ, Rohde D, Peter S. Progress in the management of advanced renal cell carcinoma (RCC). Akt Urol 2010; 41: S57-60

2 Ivy SP, Wick JY, Kaufman BM. An overview of small-molecule inhibitors of VEGFR signaling. Nat Rev Clin Oncol 2009; 6: 569-579

3 Bose D, Meric-Bernstam F, Hofstetter W et al. Vascular endothelial growth factor targeted therapy in the perioperative setting: implications for patient care. Lancet Oncol 2010; 11: 373-382

4 Ansari J, Glaholm J, McMenemin R et al. Recent advances and future directions in the management of metastatic renal cell carcinoma. Anticancer Agents Med Chem 2010; 10: $225-235$

5 Escudier B, Eisen T, Stadler WM et al. Sorafenib for treatment of renal cell carcinoma: Final efficacy and safety results of the phase III treatment approaches in renal cancer global evaluation trial.J Clin Oncol 2009; 27: 33123318

6 Motzer RJ, Hutson TE, Tomczak P et al. Overall survival and updated results for sunitinib compared with interferon $\alpha$ in patients with metastatic renal cell carcinoma. J Clin Oncol 2009; 27: 3584-3590

7 Di Lorenzo G, Buonerba C, Federico P et al. Study with sorafenib in patients with sunitinib-refractory metastatic renal cell cancer. J Clin Oncol 2009; 27:4469-4474

8 Shepard DR, Rini BI, Garcia A et.al. A multicenter prospective trial of sorafenib in patients with metastatic clear-cell renal cell carcinoma refractory to prior sunitinib or bevacizumab.J Clin Onol 2008; 26: 280 (Abstract)

9 ClinicalTrials.gov Identifier.NCT00979966

10 ClinicalTrials.gov Identifier.NCT00688753

11 ClinicalTrials.gov Identifier.NCT00720941

12 Ljungberg B, Cowan NC, Hanbury DC et al. EAU guidelines on renal cell carcinoma. The 2010 update. Eur Urol 2010; 58: 398-406

13 de Reijke TM, Bellmunt J, van Poppel $\mathrm{H}$ et al. EORTC-GU group expert opinion on metastatic renal cell cancer. Eur J Cancer 2009; 45 : 765-773

14 Rini BI, Escudier B, Tomczak P et al. Comparative effectiveness of axitinib versus sorafenib in advanced renal cell carcinoma (AXIS): a randomised phase 3 trial. Lancet 2011; 378: 1931-1939

15 Ravaud A, Digue L, Trufflandier N, Smith D. VEGFR TKI 'resistance' or transient clinical insensitivity to VEGFR TKI in metastatic renal cell carcinoma. Ann Oncol 2010; 21: 431-432

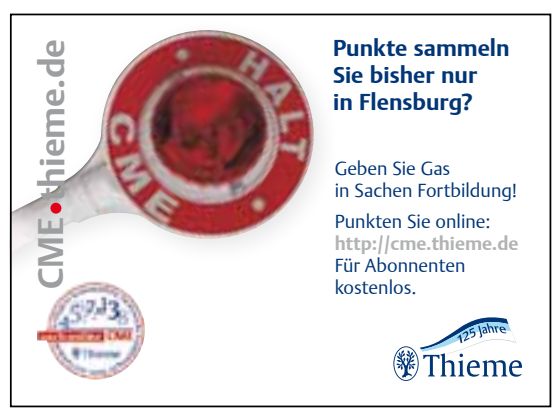

\section{Erhőht eine Vitamin-E-Zufuhr das Prostatakrebsrisiko?}

Vor nicht so langer Zeit stellte man sich die Frage: Kann Vitamin E vor Prostatakrebs schützen? Hinweise aus präklinischen und epidemiologischen Untersuchungen deuten zumindest daraufhin. Die nun von Eric A. Klein et al. veröffentlichten Nachbeobachtungsergebnisse der Studie SELECT (Selenium and Vitamin E Cancer Prevention Trial) kommen allerdings zum gegenteiligen Ergebnis.

JAMA 2011; 306: 1549-1556

\section{mit Kommentar}

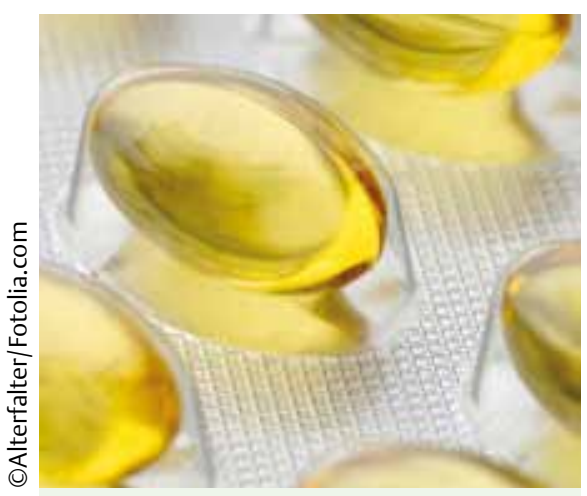

Die SELECT-Studie wurde vorzeitig abgebrochen, da das Vitamin-E-Supplement im Verdacht stand, das Prostatakrebsrisiko zu erhöhen.

SELECT ist eine 2001 initiierte, groß angelegte, doppelblinde Krebspräventionsstudie. An der Studie nahmen 35533 gesunde Männer über 50 Jahre aus den USA, Kanada und Puerto Rico teil. Die Probanden wurden auf 4 Gruppen randomisiert: Sie erhielten entweder Vitamin E (400 IU/ Tag) plus Placebo, Selen $(200 \mu \mathrm{g} / \mathrm{Tag})$ plus Placebo, eine Kombination aus beiden Präparaten oder beide Placebos. Alle Teilnehmer hatten einen PSA-Wert $\leq 4 \mathrm{ng} / \mathrm{mL}$ und einen unauffälligen rektalen Tastbefund. Die erste Interimsanalyse war für 2008 geplant (mittlere Beobachtungszeit 5,5 Jahre). Versuchspersonen, die Vitamin E zu sich genommen hatten, besaßen ein insignifikant erhöhtes Prostatakrebsrisiko. Unter Selen wurde ein leicht erhöhtes Risiko für einen Typ-2-Diabetes beobachtet. Die Studie wurde daraufhin abgebrochen, die Probanden aber bis Juli 2011 weiter verfolgt. Während dieser Zeit traten 521 weitere Prostatakarzinome auf.
Insgesamt wurden 54464 zusätzliche Personenjahre ausgewertet.

\section{Erhöhtes Krebsrisiko statt präventiver Wirkung}

Die Autoren beobachteten in allen 3 Verumgruppen ein höheres Prostatakrebsrisiko. Dies war jedoch nur in der VitaminE-Gruppe von statistischer Signifikanz. So erhöhte die Einnahme von Vitamin E das relative Risiko an Prostatakrebs zu erkranken um 17\%. Unter Selen und der Kombinationstherapie stieg das Risiko tendenziell um 9 bzw. 5\%. Der absolute Risikoanstieg war gering. Auf 1000 Personenjahre kam es unter Vitamin E zu 1,6 zusätzlichen Prostatakrebserkrankungen. Unter Selen waren es 0,8 und unter der Kombinationstherapie 0,4. Sekundäre Endpunktanalysen zeigten, dass die Einnahme von Vitamin E oder Selen weder Auswirkungen auf die Gesamtkrebsinzidenz oder die Gesamtmortalität noch auf kardiovaskuläre Ereignisse, Darm- oder Lungenkrebs hatte. Der Trend zu einem erhöhten Diabetesrisiko unter Selen bestätigte sich nicht.

\section{Fazit}

Die regelmäßige Einnahme von Vitamin $\mathrm{E}$ in einer Dosierung von $400 \mathrm{IU} / \mathrm{Tag}$ scheint bei gesunden Männern über 50 Jahren das Risiko zu erhöhen, an Prostatakrebs zu erkranken. Die Autoren warnen daher vor einem unkontrollierten Konsum von Vitamin E oder anderen rezeptfreien Nahrungsergänzungsmitteln.

Renate Ronge, Münster 
Kommentar

Vorsicht geboten bei unkritischem Einsatz

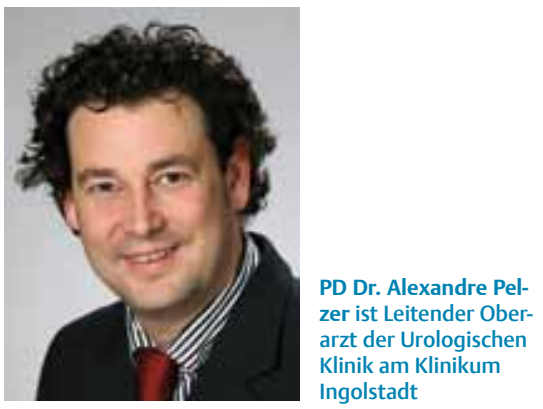

Das Prostatakarzinom als die häufigste bösartige Erkrankung von Männern fand in den letzten Jahren auch vermehrt in der Laienpresse Beachtung. Ursächlich hierfür sind nicht nur neue Behandlungsmethoden wie $z$. B. die Roboterassistierte radikale Prostataektomie oder ActiveSurveillance-Strategien, sondern auch Schwächen in der Vorsorge des Prostatakarzinoms. Abseits davon wurden schon 1994 erste Studien zur Prophylaxe des Prostatakarzinoms mit Berichten über mögliche Vorteile einer Vitamin-E-Zufuhr als Antioxidanzien zur Karzinomprophylaxe veröffentlicht. Damals gab es die Hoffnung, dass die Einnahme von Antioxidanzien und anderen Nahrungsergänzungsmitteln das Auftreten des Prostatakarzinoms reduziert und die unbe- friedigende Vorhersagekraft in der PSAbasierten Prostatakarzinomvorsorge ausgleichen kann.

\section{Heterogenität großer Studien-} populationen ist problematisch In den letzten Jahren nimmt der Verkauf sog. Nahrungsergänzungsmittel in Deutschland inflationär zu. Grundlage dafür sind Studien, insbesondere der späten 1990er-Jahre, die über positive Effekte von Antioxidanzien bei der Entstehung von bösartigen Erkrankungen berichteten. Daraufhin wurden Urologen zunehmend intensiv durch ihre Patienten mit diesem Thema konfrontiert. Dem großen Wunsch der Patienten nachkommend, nach durchgeführter Früherkennung auch wichtige Ernährungstipps auszuhändigen, konnten Kolleginnen und Kollegen nur auf präklinische oder experimentelle Daten zurückgreifen. Klein und Kollegen veröffentlichen nun eine große Beobachtungsstudie. Natürlich hat auch die vorliegende Studie positive und negative Aspekte bez. der Methodik: In der vorliegenden SELECT-Studie kommt es nun nach nochmaliger Verlängerung des Follow-up auf imposante 54464 Patientenjahre auf nicht nur ernüchternde, sondern v.a. auf warnende Zahlen. Neben der ernormen Patientenanzahl sind jedoch, ähnlich anderer Großstudien, die Heterogenität der Studienteilnehmer und des Studienprotokolls Kritikpunkte. Aussagen über die Überprüfung des Patientenkollektivs bez. der Compliance bei der Vitamin-E- oder Selen-Einnahme fehlen ebenso wie Angaben über die Methodik der Prostatakarzinomdiagnostik. Nicht beachtet wurden auch andere Einflussfaktoren der Studie wie die mögliche familiäre Vorbelastung der Patienten. Trotzdem versucht die Studie mit großer Sorgfalt und genauen Patientenvisiten und Protokollen mit aufwendigen statistischen Mitteln eine möglichst homogene Aussage zu treffen. Dies gelingt ihr mit dem deutlichen Hinweis durch das wichtige Endergebnis: Männer mit vermehrter VitaminE-Zufuhr hatten ein signifikant erhöhtes Risiko an einem Prostatakarzinom zu erkranken.

\section{Hilft nicht und schadet noch dazu}

Mit einem Vergleich der untersuchten Studienmedikamentendosis mit den in Deutschland erhältlichen Dosierungen in frei verkäuflichen Mengen werden wir uns schwer tun - die Frage nach dem Sinn von Vitamin-E zur Prostatakarzinomprophylaxe scheint trotzdem beantwortet: Urologen sollten ihren Patienten dringend davon abraten, zusätzliche Vitaminpräparate einzunehmen. Der Schaden ist höher als der Nutzen und der Spruch „Hilft es nichts, schadet es wenigstens nichts" hat seine Ungültigkeit bewiesen.

\section{PD Dr. Alexandre Pelzer, Ingolstadt}

Forschungspreis Prostatakarzinom

\section{Bewerbung nicht} vergessen!

Der „Forschungspreis Prostatakarzinom“ fördert junge Nachwuchswissenschaftler aus Klinik oder Praxis, die sich in ihrer Arbeit mit Fragen zur Entstehung, Diagnose und Therapie des Postatakarzinoms beschäftigen. Gestiftet wird der mit $10000 €$ dotierte Preis vom Münchener Unternehmen Astellas Pharma.

„Wissenschaft, Fortschritt, Leben“ - unter diesem Motto steht die 64. Jahrestagung der Deutschen Gesellschaft für Urologie. Es gilt neue Talente zu gewinnen und $\mathrm{zu}$ fördern, ebenso wie die in der Wissenschaft tätigen Kollegen zu unterstützen. Hinter diesem Vorhaben steht auch der „Forschungspreis Prostatakarzinom“. Mit der Vergabe setzt sich die DGU für wissen- schaftliche Forschungsprojekte ein. „Es werden stetig talentierte Nachwuchskräfte benötigt, um die Qualität in der Urologie aufrechtzuerhalten. Mit dem „Forschungspreis Prostatakarzinom“, der dieses Jahr zum zweiten Mal verliehen wird, sollen diese entdeckt und deren Forschungsvorhaben unterstützt werden“, sagt DGU-Kongresspräsident Prof. Stefan C. Müller.

\section{Bewerbungunterlagen}

Bewerben können sich Wissenschaftler bis einschließlich zum 40. Lebensjahr mit Forschungsstandort in Deutschland. Für die Teilnahme ist ein Projektplan zu erstellen, der Ziele, Methoden und Ressourcen enthält sowie eine Zeitplanung und eine Zusammenfassung. Das Forschungsprojekt darf noch nicht abgeschlossen oder veröffentlicht sein. Die ausführlichen Teilnahmebedingungen finden Sie unter Wissenschaftliche Preise auf:

\section{http://www.dgu-kongress.de}

Die Bewerbungsfrist endet am 15. Juni 2012. Die Verleihung des Forschungspreises findet auf der diesjährigen Jahrestagung der Deutschen Gesellschaft für Urologie vom 26.-29. September 2012 in Leipzig statt.

\section{Nach einer Pressemitteilung (Astellas Pharma)}

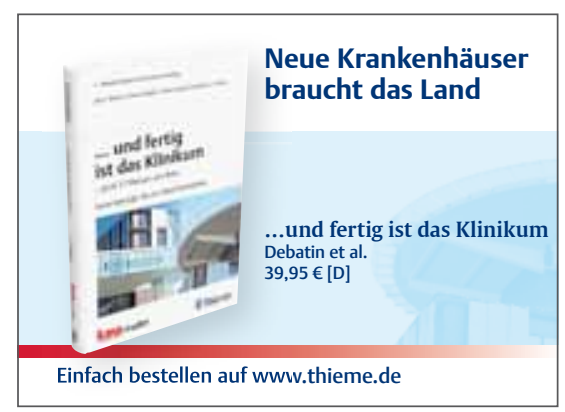




\section{Intensive Urotherapie wirkt bei Kindern nachhaltig}

Die konservative Urotherapie als Behandlungsoption funktioneller Inkontinenz bei Kindern beinhaltet u.a. die Beratung bez. des Miktionsverhaltens, das Miktions- und Biofeedbacktraining oder die Physiotherapie. Einzelne Studien belegen, dass eine stationäre Urotherapie bei ca. 70\% der Kinder erfolgreich ist. Ob und wie lange dieser Effekt anhält, hat nun eine niederländische Studie untersucht. J Urology 2011; 78: 1391-1396

\section{mit Kommentar}

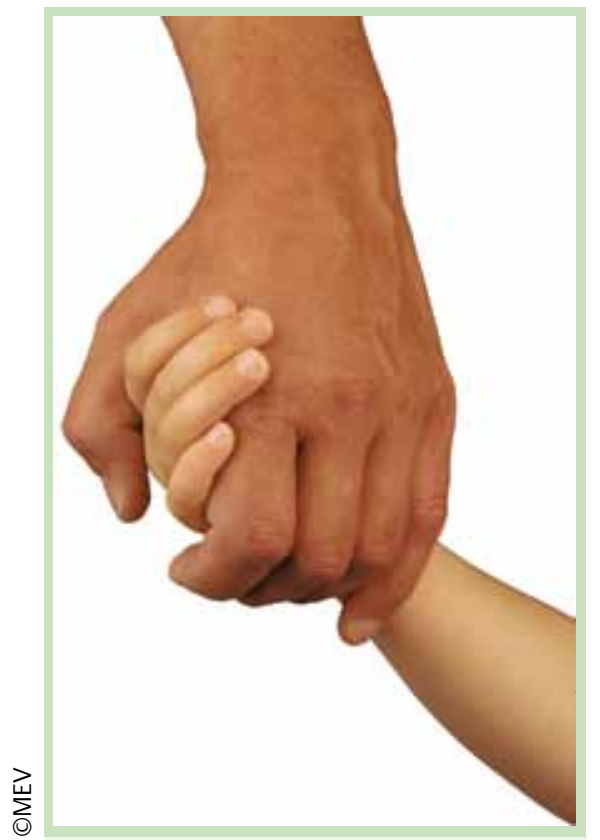

Kinder mit funktioneller Inkontinenz profitieren von einer frühen, intensiven Urotherapie, so das Ergebnis dieser niederländischen Studie. Die älteren Kinder sprachen zwar schneller auf die Therapie an, im Langzeitverlauf machte das Alter der Patienten jedoch keinen Unterschied.

Wer in der Kindheit gut auf eine intensive Urotherapie anspricht, hat auch langfristig i.d.R. keine Miktionsbeschwerden. Zu diesem Ergebnis kamen Marianne Vijverberg, University Medical Center Utrecht/ Niederlande, und Kollegen anhand einer Kohortenstudie mit 75 Patienten zwischen 6 und 17 Jahren. Diese wurden zwischen 1986 und 1990 wegen Blasenentleerungsstörungen oder überaktiver Blase stationär urotherapeutisch behandelt. Der Altersdurchschnitt der Kinder lag zu
Beginn der Studie bei 9,9 $\pm 2,4$ Jahren. Die Patienten erhielten in einem intensiven, stationären Training Anleitungen zu Miktionsgewohnheiten wie Häufigkeit des Wasserlassens und der Sitzpositionen beim Toilettengang. Zudem wurde geschult, wie Obstipation und Harnwegsinfektionen verhindert werden könnten. Die Urotherapie beinhaltete auch die Untersuchung des Harnflusses durch Uroflowmessung, Biofeedback-Training und Anleitungen zur Verhaltensänderung.

\section{4\% der Patienten erzielten gute Langzeitresultate $\nabla$}

Nach 6 Monaten wurden die Trainingsergebnisse anhand der folgenden Variablen gewertet: Art des Harndrangs, Einnässen am Tag, Miktionsfrequenz, Harndrang und Miktionsprofil. Dieselben 4 Variablen wurden für die Langzeitstudie im Jahr 2007 (durchschnittlich 17,9 Jahre später) erneut telefonisch abgefragt. Die Bewertung erfolgte jeweils anhand der Kategorien „gut", „moderat“ und „schlecht“. Die Kurzzeitergebnisse unterschieden sich nicht signifikant von denen der Langzeitbeobachtung $(p=0,726)$. Von den 56 Patienten, die damals ein gutes Resultat erzielten, fielen 47 Patienten im Langzeitverlauf weiterhin in die Kategorie "gut“. Von 7 Patienten mit zunächst „moderatem" Erfolg konnten sich 5 Patienten zu einem „gut" steigern. Auch von den $12 \mathrm{~Pa}-$ tienten, deren Ergebnisse ursprünglich „schlecht“" ausfielen, verbesserten sich 11 Patienten zu einem „gut“. Im Durchschnitt waren diese 16 Patienten, die sich im Verlauf verbessert hatten, 2,1 Jahre nach der intensiven Urotherapie symptomfrei. Insgesamt wiesen $84 \%$ der Patienten im Langzeitverlauf gute Resultate auf. Dennoch waren zum Zeitpunkt der Langzeitbeobachtung bei 8 Patienten die Werte noch immer „mangelhaft", bei 4 Patienten sogar „schlecht“. Die Autoren untersuchten außerdem, inwiefern das Alter der Patienten bei Therapiebeginn die Wirksamkeit der Urotherapie beeinflusste. Obwohl ältere Kinder nach 6 Monaten offensichtlich bessere Resultate erzielten, war dieser Effekt im Langzeitverlauf nicht mehr nachweisbar $(p=0,687)$.

\section{Fazit}

Die Studie liefere mehrere Argumente, funktionelle Störungen des unteren Harntraktes schon im Kindesalter urotherapeutisch zu behandeln, so die Autoren. Zwar wurde ein Großteil derjenigen Patienten, die kurzfristig höchstens moderat auf die Urotherapie ansprachen, im späteren Verlauf auch spontan symptomfrei, aber trotzdem sei Abwarten keine Option, weil sonst behandelbare Ursachen übersehen werden könnten. Zudem verbessere die Urotherapie die Lebensqualität der Patienten, da Inkontinenz im Kindesalter einen negativen psychologischen Effekt habe. Die Autoren sehen zudem einen finanziellen Vorteil frühzeitiger urotherapeutischer Behandlung: Die Kosten für Arztbesuche und Medikamente fielen ansonsten über die Jahre höher aus.

Dr. Bettina Rakowitz, Sachsen b. A.

Kommentar

Erste Ergebnisse zu
Langzeitrespondern

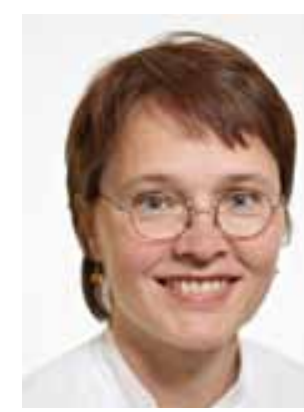

Dr. Cornelia Möhring ist Leiterin der Kinderurologie an der Klinik für Urologie, Kinderurologie und Urologische Onkologie des Klinikums Niederberg, Velbert

Die Arbeitsgruppe um Frau Vijverberg publizierte 2011 Daten einer LangzeitAnalyse zu einer eigenen Studie aus dem Jahr 1997 im Journal of Urology [1]. Hierbei handelte es sich ursprünglich um eine 
Auswertung eines stationären Trainingsprogramms für Kinder mit funktionellen Blasenentleerungsstörungen. 1997 wurden 95 Kinder (Alter: 6-17 Jahre) in einem 10-tägigen stationären Aufenthalt einer Urotherapie inkl. Biofeedback zugeführt. Die Autoren schlossen nur Kinder ein, die unter einer Urge-Inkontinenz litten und zuvor therapierefraktär über 12 Monate hinweg medikamentös und mit ambulanter Urotherapie behandelt wurden.

In der aktuellen Arbeit wurden aus diesem Patientenkollektiv erneut 75 Patienten rekrutiert und in einem Follow-up von minimal 16,2 Jahren erneut befragt. Die kindliche LUTS spielt im pädiatrischen Alltag mit einer Prävalenz von $10 \%$ bei Kindern unter 7 Jahren und deutlich belastenden Symptomen für Kind und Eltern eine wesentliche Rolle. Einnässen ist das häufigste urologische Symptom im Kindesalter. Es ist für den behandelnden Arzt eine Herausforderung, da der Erfolg oder Misserfolg der eingeleiteten Therapie das psychische und auch das körperliche Wohlergehen des Kindes deutlich beeinflusst. Daher sind Erkenntnisgewinne in der Langzeittherapie für die meisten kinderärztlich tätigen Ärzte durchaus von Relevanz.

Frau Vijverberg und Kollegen zeigten zum ersten Mal in diesem Kontext der kindlichen LUTS bei zunächst therapierefraktären Patienten die Ergebnisse einer stationären Urotherapie über ein mindestens 16 Jahre langes Follow-up. Die Literatur bot bis zu diesem Zeitpunkt nur einen Überblick über maximal 5 Jahre. Die Prävalenz der kindlichen LUTS liegt bei $10 \%$ bei Kindern unter 7 Jahren und persistiert noch bei $1-2 \%$ bis zum 18 . Lebensjahr und wird im kurzen Follow-up mit einer Heilungsrate von $74 \%$ betrachtet. Es existieren nur wenige Daten zu Langzeitrespondern auf die Urotherapie (exkl. Biofeedback) durch Wiener et al. 2000 mit einer Rate von 59,4\% [2]. Langzeitresponder auf die Urotherapie (inkl. Biofeedback) wurden bis dato nicht klinisch belegt.

Die Studie von Vijverberg et al. besticht durch ein sehr langes Follow-up von 16,221,8 Jahren und bietet so sicher ein hohes Maß an Validität für den Erfolg der Urotherapie in der Behandlung der kindlichen LUTS. Auch die erreichte Rücklaufquote von rund 78\% (75 Kinder von 95) in der Langzeit-Patientenpopulation ist für eine angemessene Objektivität und Reliabilität mehr als ausreichend. In der Literatur sind solche qualitativen Gütekriterien sicher einzigartig und unterstreichen die Ergebnisse.

Anzumerken bleibt, dass ein Erfolg der LUTS-Therapie bei Vijverberg et al. lediglich an 4 Variablen gemessen wurde (Inkontinenzereignisse/Tag, Miktionsfrequenz, Drangereignisse und Harnstrahlprojektion). Unklar blieb hierbei, ob ein Flow-EMG durchgeführt wurde, ob Harnwegsinfektionsrezidive aufgetreten waren und ob einige der Kinder Restharnbildungen gezeigt hatten. Davon abgesehen, ist eine stationäre Urotherapie - zumindest im deutschsprachigen Raum - aus Kostengründen nur noch in Einzelfallentscheidungen zu realisieren. Da diese stationäre Urotherapie aktuell nicht praktikabel erscheint, fällt es schwer, diese Ergebnisse banal auf eine ambulante Urotherapie zu übertragen.

Die Erfolgsrate dieser Studie deckt sich mit den bisher publizierten Daten und kann mit $60 \%$ aus dem realen Patientengut abgeleitet werden. Trotzdem ist sicher ein Kritikpunkt, dass hier spezifische Erfolgsvariablen gewählt wurden, die einen Vergleich mit anderen Arbeiten nicht möglich macht. Wie Flow-EMG-Kurven bei Kindern, die an LUTS leiden, Jahre nach einem Biofeedback-Training aussehen, bleibt weiterhin eine Frage von Interesse, die Vijverberg et al. zurzeit nicht beantworten können. Hier würden weiterführende Studien für Klarheit sorgen. Des Weiteren würde eine Langzeitstudie zum ambulanten Biofeedback-Training für unsere kinderurologische Sprechstunde von ungleich größerer Bedeutung sein.

\section{Dr. Cornelia Möhring, Velbert}

\section{Literatur \\ 1 Vijverberg MAW, Elzinga-Plomp A, Messer AP et al. Bladder rehabilitation, the effect of a cognitive training programm on urge in- continence Eur Urol 1997; 31: 68-72 \\ 2 Wiener JS, Scales MT, Hampton J et al. Long- term efficacy of simple behavioral therapy for daytime wetting in children.J Urol 2000; 164: 786-790}

Praxishandbuch Männergesundheit: Interdisziplinärer Beratungs- und Behandlungsleitfaden Harth, Wolfgang; Brähler, Elmar; Schuppe, Hans-Christian (Hrsg.) MWV, Berlin 2012; ISBN 9783941468511

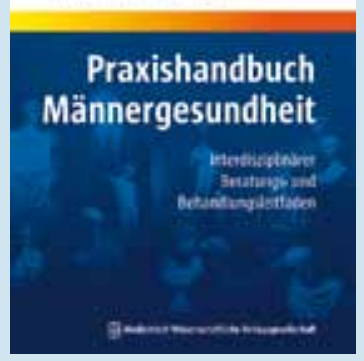

Das Praxishandbuch Männergesundheit beleuchtet zahlreiche andrologische Facetten. Medizinische Aspekte diverser Disziplinen, aber auch psychosoziale Fragestellungen und komplementäre Ansätze werden auf 440 Seiten in einzelnen Kapiteln besprochen. So ergibt sich ein interessantes Mosaik und eine kurzweilige Lektüre. Klassische urologische Krankheitsbilder wie die Erektile Dysfunktion, das Prostatakarzinom oder Hodentumoren werden ebenso erläutert wie weniger geläufige Problemstellungen, z.B. Somatoforme Störungen, Steroidmissbrauch, Burn-out und Männerkosmetik.
Das Buch bietet einen abwechslungsreichen Blick auf zahlreiche andrologische Themen und eignet sich deshalb sowohl als Lehrbuch, als auch zum Nachschlagen einzelner Problemfelder. Besonders interessant sind dabei die Nischen, die weniger geläufig sind. Es ist für die tägliche klinische Arbeit sicher nicht notwendig, aber dennoch spannend, z.B. über die Auswirkungen des 2. Weltkriegs auf die Psyche der betroffenen Männer zu lesen.

Den Autoren ist mit dem Praxishandbuch Männergesundheit eine empfehlenswerte Zusammenstellung zahlreicher Aspekte des andrologischen Themenkreises gelungen, das besonders durch die Besprechung von weniger beachteten Problemstellungen überzeugt.

Matthias Schweizerhof, Mainz 


\section{Laserakupunktur ist der Scheinbehandlung überlegen}

Die primäre monosymptomatische Enuresis nocturna (MNE), das nächtliche Einnässen ohne Miktionsauffälligkeiten oder Blasenfunktionsstörungen, betrifft ca. $15 \%$ der Kinder > 5 Jahre. Die Behandlungsansätze sind vielschichtig. Ob die Laserakupunktur eine weitere Therapieoption bietet, hat nun eine türkische Studie untersucht. J Urol 2011; 185: 1852-1856

\section{mit Kommentar}

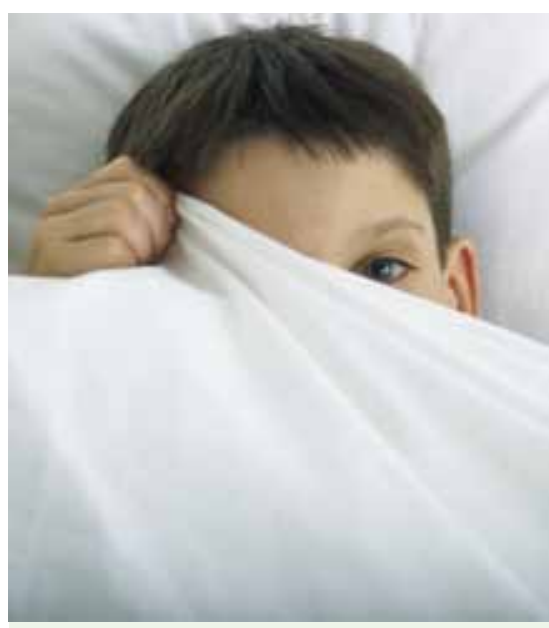

In der aktuellen Studie reduzierte die Laserakupunktur bei Kindern mit primärer monosymptomatischer Enuresis nocturna die wöchentlichen Einnässepisoden. Nach 6 Monaten waren 54\% der laserakupunktierten Kinder vollständig trocken, in der Placebogruppe nur $12 \%$ der Patienten (@PhotoAlto/Symbolbild).

M. Ihsan Karaman, Haydarpasa Numune Training and Research Hospital, Istanbul/ Türkei, und Kollegen schlossen 53 Jungen und 38 Mädchen $(n=91)$ im Alter von 5-16 Jahren mit MNE in ihre prospektive Studie ein. Sie randomisierten die medikamentös nicht vorbehandelten Kinder in 2 Gruppen: Die Verumgruppe $(n=62)$ wurde über 4 Wochen 3-mal wöchentlich mit Laserakupunktur behandelt. In der Placebogruppe $(n=29)$ kam ein Schein-Laser in den 12 Sitzungen zum Einsatz. In der Verumgruppe brachen 5 Kinder die Studie vorzeitig ab, in der Placebogruppe 3.

Die Autoren dokumentierten die Anzahl der wöchentlichen Einnässepisoden vor der Akupunkturbehandlung und bis zu 180 Tage danach. Traten im Anschluss an die Therapie keine enuretischen Episoden mehr auf, galten die Kinder als vollständig trocken. Ein Rückgang der Enuresisfrequenz um mindestens die Hälfte wurde als partielle Besserung bewertet.

\section{Hälfte der laserakupunktierten Kinder nach 6 Monaten trocken $\nabla$}

Die Zahl der wöchentlichen Enuresisepisoden war 6 Monate nach der Akupunkturbehandlung von durchschnittlich 4,3 auf 1,7 gesunken. In der Placebogruppe gingen die Episoden von 4,1 auf 3,1 zurück. Bei den Kontrolluntersuchungen nach 15, 30, 90 und 180 Tagen waren 40, 56, 56 und 54\% der laserakupunktierten Kinder vollständig trocken. In der Placebogruppe waren es $8 \%$ nach 15 Tagen und $12 \%$ nach jeweils 30, 90 und 180 Tagen. Eine partielle Besserung erzielten 32, 21, 21 und $23 \%$ der Kinder im Verumarm und 4, 4, 8 und $8 \%$ der Kinder im Placeboarm nach 15, 30, 90 und 180 Tagen. Insgesamt war die Laserbehandlung der Scheinakupunktur signifikant überlegen. Bei einem Kind der Lasergruppe kam es nach 6 Monaten zu einem Rückfall.

\section{Fazit}

Die Laserakupunktur biete eine nichtinvasive, leicht anwendbare, schmerzlose und kostengünstige Alternative zur Behandlung der MNE, so die Autoren. Weitere Studien mit einer höheren Anzahl an Patienten seien jedoch nötig, um die Wirkung zu bestätigen.

Renate Ronge, Münster

\section{Kommentar}

\section{Eine mögliche Behandlungsoption für die Praxis?}

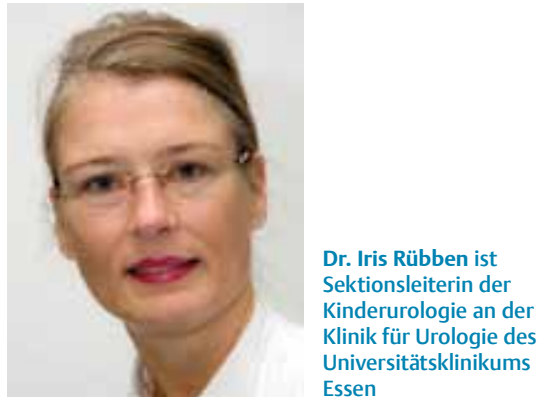

Die monosymptomatische nächtliche Einnässsymptomatik (MNE) hat eine Prävalenz von $5-10 \%$ bei 7 -jährigen Kindern, d.h. sie ist ein relevantes Problem, welches andererseits aber auch mit einer hohen spontanen Heilungsrate von 15\% pro Jahr einhergeht. Gemäß der neuen Nomenklatur umfasst die MNE nächtlichen unbemerkten Harnverlust, ohne dass eine Blasenentleerungsstörung, d.h. eine Tagessymptomatik, nachgewiesen werden kann. Die 2 allgemein anerkannten Behandlungsoptionen der MNE (Evidenz Grad A) sind die medikamentöse Therapie mit Desmopressin oder verhaltenstherapeutische Maßnahmen mit einem Alarmsystem. Beide Behandlungsoptionen erreichen in 50-70\% einen Heilerfolg in unselektierten Patientenpopulationen.

Immer wieder wird über alternative Behandlungsoptionen berichtet, insbesondere über die Akupunktur als nichtinvasive und nahezu nebenwirkungsfreie Behandlungsmethode. Auch Eltern wünschen in der Behandlung ihrer Kinder oft ein alternatives Behandlungsangebot, sodass die Akupunktur bei Kindern mit MNE regelmäßig Gegenstand klinischer Beratungsgespräche ist.

Die Autoren initiierten eine prospektiv randomisierte, placebokontrollierte einseitig verblindete Studie, in die 91 Kinder (mittleres Alter 8,6 Jahre) ohne medikamentöse Vorbehandlung eingingen (53 Knaben, 38 Mädchen). Es wurden 2 Gruppen gebildet, die eine erhielt eine Laserakupunktur, die andere eine Non-LaserLichtquellenbehandlung. Eine Evaluation, die ein Einnässtagebuch umfasste, erfolgte nach 15, 30, 90 und 180 Tagen, wobei gezeigt wurde, dass die Kinder, die durch Laserakupunktur behandelt wurden, be- 
reits nach 30 Tagen eine signifikant bessere Trockenheits-/Heilungsrate von 56\% gegenüber der nicht Laser-behandelten Gruppe von nur 12 \% aufwiesen. Die Heilungsrate war relativ konstant, sodass nach 180 Tagen noch $54 \%$ der Kinder vollständig trocken waren. Im Gegensatz dazu wurden in der nicht behandelten Gruppe nur 12\% (3 Kinder) vollständig trocken.

\section{Schwächen der Arbeit}

Als Ätiologiefaktoren für das Auftreten einer MNE werden eine reduzierte funktionelle Blasenkapazität oder eine hohe nächtliche Harnproduktion - verbunden mit einer nicht adäquaten, nächtlichen Perzeption des Harndrangs - diskutiert. Als besondere Schwäche der Arbeit muss angemerkt werden, dass die in die Studie eingebrachten Kinder nicht weiter bez. ihrer funktionellen Blasenkapazität evaluiert wurden. Der Messparameter der Studie war lediglich die Anzahl trockener Nächte.

Im selben Heft des „Journal of Urology“, in der die o. g. Arbeit von Karaman und Mitarbeitern erschienen ist, wurde auch die Arbeit von Radvanska und Mitarbeitern (Arbeitsgruppe um Søren Rittig, Aarhus/ Dänemark) mit selbigem Thema publiziert [1]. Auch diese Arbeitsgruppe hat eine prospektiv randomisierte, einseitig verblindete und placebokontrollierte Studie durchgeführt, in der der Effekt der Laserakupunktur bei Kindern mit nächtlicher Einnässsymptomatik evaluiert wurde. Im Gegensatz zu der begutachteten $\mathrm{Ar}$ beit von Karaman und Mitarbeitern kommt die Arbeitsgruppe zu einem ganz anderen Ergebnis. Bei vergleichbarem mittleren Alter wurden hier mit 31 Kindern mit MNE zwar deutlich weniger Patienten in die Studie eingebracht, nach einem 5-wöchigen Laserakupunktur-Behandlungsintervall besserte sich die Einnässsymptomatik jedoch nicht signifikant. In dieser Studie wurde zusätzlich die funktionelle Blasenkapazität durch ein Blasentagebuch evaluiert, welches die Einzelharnmengen (in $\mathrm{ml}$ ) protokollierte.

In einem systematischen Review von Bower et al. (Arbeitsgruppe C.K. Yeung, Hong Kong) über Akupunkturbehandlungen bei Kindern mit nächtlicher Einnässsymptomatik wurden in sehr aufwendiger Recherche und Auswahlmethodik sämtliche Arbeiten zu diesem Thema verglichen [2]. Als Ergebnis kann lediglich ein vorsichtiger Trend erkannt werden: Die Laserakupunktur könnte in der Behandlung der MNE effektiv sein.

\section{Fazit}

Bisher gibt es zur Akupunkturbehandlung bei Kindern mit monosymptomatischer nächtlicher Enuresis sehr widersprüchliche Daten. In manchen Studien wird eine Ansprechrate von 50\% und mehr erzielt. So konnte die Studie von Karaman et al. eine Trockenheitsrate $>50 \%$ in der Behandlungsgruppe mit signifikantem Ansprechen im Vergleich zur Placebogruppe nachweisen, während die Arbeitsgruppe aus Aarhus mit ähnlichem Studiendesign kein Ansprechen ergab [1]. Die Ergebnisse reflektieren Arbeiten zu diesem Thema der vergangenen Jahre - auch zu erwähnen die Arbeit von Radmayr und Mitarbeitern, Innsbruck, die die Akupunkturbehandlung mit der Desmopressin-Medikation verglichen und vergleichbare Ansprechraten größer $50 \%$ berichten konnten [3].

Ich möchte mich daher einem Kommentar von Herrn Nevéus anschließen, dass die Akupunkturbehandlung sicher eine spannende Therapiealternative bleibt, dass aber bez. einer allgemeinen Therapieempfehlung ihr Effekt in größeren Studien fundierter untersucht werden muss [4]. Da die Therapie insgesamt keine besonderen Nebenwirkungen in bisher allen Untersuchungen gezeigt hat, kann eine individuelle Handlungsentscheidung nur vom behandelnden Arzt und den Eltern getroffen werden.

Literatur

1 Radvanska E, Kamperis K, Kleif A et al. Effect of Laser Acupuncture for Monosymptomatic Nocturnal Enuresis on Bladder Reservoir Function and Nocturnal Urine Output. J Urol 2011; 185: 1857-1862

2 Bower WF, Diao M, Tang JL et al. Acupuncture for nocturnal enuresis in children: A systematic review and exploration of rationale. Neurourol Urodyn 2005; 24: 267-272

3 Radmayr C, Schlager A, Studen M, et al. Prospective randomized trial using laser acupuncture versus desmopressin in the treatment of nocturnal enuresis. Eur Urol 2001; 40: 201-205

4 Nevéus T. Editorial Comment, J Urol 2011; 185: 1862

\section{Prostatakarzinom}

\section{Strahlenbehandlung in allen Stadien hoch effektiv}

Die besten Behandlungsergebnisse beim lokalisierten Prostatakarzinom werden mit einer Strahlentherapie erreicht - entweder als Brachytherapie oder als Kombination von Brachytherapie und externer Strahlentherapie. Darauf wies die Deutsche Gesellschaft für Radioonkologie (DEGRO) anlässlich einer kürzlich im British Journal of Urology International (BJUI) publizierten, systematischen Metaanalyse von mehr als 52000 Erkrankungsfällen hin.
Das Prostatakarzinom ist mit weit über 60000 Neuerkrankungen in Deutschland jährlich der häufigste maligne Tumor und die dritthäufigste krebsbedingte Todesursache beim Mann. Durch stetig verbesserte Diagnoseverfahren werden heutzutage mehr als 70\% aller Prostatakarzinome relativ früh entdeckt. Oft sind diese lokal begrenzt, und damit bestehen gute Heilungschancen. Nicht jeder Patient benötigt eine Behandlung, bei einigen ist eine regelmäßige sog. aktive Überwachung die beste Option. Wenn jedoch eine lokale Behandlung des Prostatatumors indiziert ist, kommen verschiedene Verfahren in Betracht: die Radikaloperation oder verschiedene Strahlentherapie-Verfahren, darunter auch die Brachytherapie (permanente Seed-Implantation oder HDRBrachytherapie).
Prof. Jürgen Dunst, Präsident der DEGRO und Direktor der Klinik für Strahlentherapie an der Universität Lübeck, betont: „Operation und Strahlentherapie gelten als gleichwertig. Bisher gibt es jedoch keine randomisierten Studien mit einem direkten Vergleich der Verfahren." Daher begrüßt Dunst die jüngst veröffentlichten Ergebnisse einer internationalen Gruppe um Dr. Peter Grimm, Seattle. Alle in der Fachliteratur veröffentlichten Behandlungsergebnisse der letzten Jahre (20002010) wurden in dieser Metastudie systematisch analysiert. Untersucht wurden die Behandlungsergebnisse nach

- Radikaloperation (16697 Patienten, davon 1381 mit robotergestützter OP), - interstitieller Brachytherapie (insgesamt 22479 Patienten, zum Teil mit 


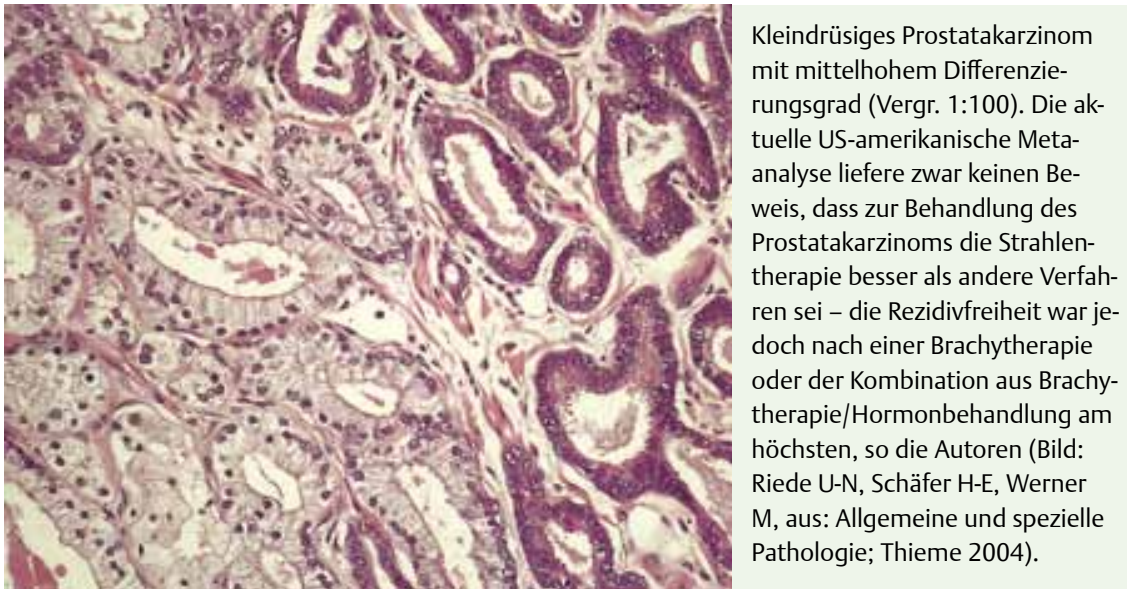

zusätzlicher externer Bestrahlung und Antihormontherapie),

- alleiniger externer Bestrahlung

(12 082 Patienten) oder

- anderen Verfahren (532 Patienten mit hochfokussiertem Ultraschall, $227 \mathrm{~Pa}-$ tienten mit Kryotherapie).

Prof. Thomas Wiegel, Ärztlicher Direktor der Abteilung Strahlentherapie am Universitätsklinikum Ulm und Beauftragter der DEGRO für Prostata-Zentren, erklärt: „Als Maß für die Wirksamkeit der Behandlung wurde die biochemische Rezidivfreiheit analysiert, also der Anteil von Patienten, bei denen sich der Wert des prostataspezifischen Antigens nach der Behandlung nicht verschlechterte.“

Alle Patienten seien auf der Basis der vor Therapiebeginn vorliegenden Befunde in drei Risikogruppen aufgeteilt worden.
Wiegel fasst die Ergebnisse zusammen: „Diese Studie liefert zwar keinen Beweis, dass eine bestimmte Therapie eindeutig besser ist als andere. Die biochemische Rezidivfreiheit war in dieser Analyse aber nach einer Brachytherapie oder nach einer Kombination aus Brachytherapie und/ oder externer Bestrahlung und Hormontherapie am höchsten. StrahlentherapieVerfahren sind in allen Krankheitsstadien eine hocheffektive Behandlung und im Vergleich zu einer Radikaloperation mindestens gleichwertig oder besser." Diese Analyse stelle eine wichtige Hilfe für die Entscheidungsfindung von Ärzten und Patienten dar.

\section{Nach einer Pressemitteilung \\ (Deutsche Gesellschaft für \\ Radioonkologie e.V.)}

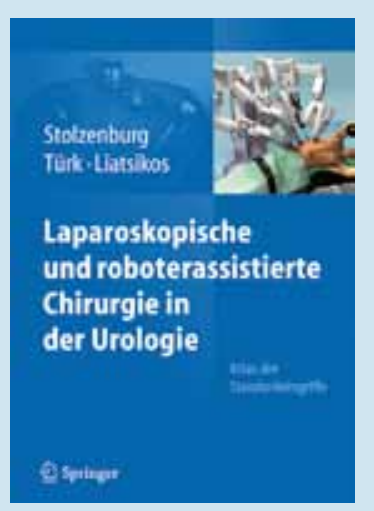

Laparoskopische und roboterassistierte Chirurgie in der Urologie: Atlas der Standardeingriffe Stolzenburg, Jens-Uwe; Türk, Ingolf; Liatsikos, Evangelos N. (Hrsg.) Springer, Berlin/Heidelberg 2011; ISBN 9783642103780

Die Herausgeber Stolzenburg, Türk und Liatsikos haben mit diesem Buch einen Atlas der minimalinvasiven chirurgischen Eingriffe in der Urologie verfasst. Es beschreibt detailliert und reich bebildert alle urologischen Operationen, die laparoskopisch oder minimalinvasiv durchgeführt werden können. Dabei werden die relevanten Schritte vom Stechen der Trokare bis zu den entscheidenden Momenten der Operation mit jeweils einem Bild bedacht und in einem Begleittext erläutert. Die Bilder sind verständlich und erklärend und geben den Situs adäquat wieder. Im Text werden einzelne Schritte beschrieben, anatomische Leitstrukturen angegeben und auch die Handgriffe des Assistenten kommentiert. Die Zahl der Bilder ist ausreichend, um die operativen Schritte verstehen und nachvollziehen zu können.

Den Autoren ist es gelungen, einen gut verständlichen und anschaulichen Operationsatlas zu den laparoskopischen und roboterassistierten Eingriffen in der Urologie zu verfassen. Das Buch wendet sich einerseits an den Anfänger, dem die Assistenz einer Operationen klarer wird, aber auch an den noch wenig erfahrenen Operateur, der einen klaren Leitfaden an die Hand bekommt, mit dem er die Operation leichter meistern kann. Ob der Atlas dem erfahrenen Operateur Neues verrät, sei dahingestellt, aber für den wenig erfahrenen Chirurgen ist dieses Buch mit einem Preis von 129,95 € eine lohnende Anschaffung.

Dr. Sebastian Nestler, Mainz 